06,13

\title{
Особенности сегнетоэлектрического состояния в двухслойных гетероструктурах на основе титаната бария-стронция
}

\author{
(ㄱ Д.В. Стрюков, В.М. Мухортов, Ю.И. Головко, С.В. Бирюков \\ Южный научный центр РАН, \\ Ростов-на-Дону, Россия \\ E-mail: mukhortov1944@mail.ru
}

(Поступила в Редакцию 7 мая 2017 г.

В окончательной редакции 21 июня 2017 г.)

\begin{abstract}
Исследованы структурные свойства однослойных и двухслойных гетероструктур на основе пленок титаната бария-стронция различного состава, осаженных по механизму Франка-Ван дер Мерве на подложку оксида магния. Гетероструктуры созданы ВЧ-распылением стехиометрических керамических мишеней в распылительной системе „Плазма 50 СЭ“. Принципиальное отличие данного способа осаждения от известных аналогов состоит в том, что рост монокристаллических пленок происходит из дисперсной фазы оксида, образующейся в плазме сильноточного высокочастотного разряда при распылении керамической мишени на кластерном уровне. Установлены особенности проявления сегнетоэлектрического состояния в двухслойных гетероструктурах при изменении порядка следования пленок с различным составом титаната бария-стронция.
\end{abstract}

Исследование выполнено в рамках реализации Государственного задания на 2017 г. (проект № 0120-1354-247), а также при поддержке РФФИ (грант № 16-29-14013).

DOI: 10.21883/FTT.2018.01.45297.186

\section{1. Введение}

Сегнетоэлектрические гетероструктуры, состоящие из слоев различного состава (многослойные и сверхрешетки), сформированные на диэлектрических подложках, интенсивно исследуются [1-3] с целью повышения диэлектрической проницаемости [4] и ее термостабильности, понижения диэлектрических потерь и увеличения диэлектрической нелинейности под действием внешнего электрического поля для задач сверхвысокочастотной микроэлектроники [5-8]. Исследования направлены на поиски новых путей управления свойствами гетероструктур за счет деформации элементарной ячейки в тонкой пленке и интерфейсного влияния $[5,6]$. Деформация ячейки вызывается несоответствием параметров решетки подложки и пленки и различием их коэффициентов теплового расширения. Управление деформацией элементарной ячейки в гетероструктурах в настоящее время проводится подбором материала пленки и подложки или созданием буферного слоя. Развитие последнего трансформировалось в новое направление - создание многослойных гетероструктур. Именно таким образом было достигнуто уменьшение токов утечки в сегнетоэлектрических элементах за счет создания многослойных гетероструктур сегнетоэлектрикдиэлектрик [9]. Тем не менее, исследования двухслойных пленок $\mathrm{Ba}_{x} \mathrm{Sr}_{(1-x)} \mathrm{TiO}_{3}$ с различным значением $x$ не привели к ожидаемой суперпозиции температурной зависимости диэлектрической проницаемости в таких гетероструктурах по сравнению с однослойными [10]. Одним из направлений, связанным с поисками путей эффективного управления свойствами гетероструктур на основе сегнетоэлектриков, является „доменная инженерия“ - управление свойствами через изменение доменной конфигурации под действием двумерных напряжений [4,11-13]. Возникновение спонтанной деформации в результате первого фазового перехода после осаждения и формирование доменной структуры естественно влияет на конечные двумерные деформационные поля. Вопросу эффективности данного механизма и посвящено наше исследование. Влияние на свойства формирующейся после осаждения пленки доменной структуры и ее вклад в деформацию элементарной ячейки исследовались нами на примере двухслойников $\mathrm{Ba}_{x} \mathrm{Sr}_{(1-x)} \mathrm{TiO}_{3}$ с $x=0.8$ и $x=0.4$ (ВST08, ВST04 соответственно) при использовании различной последовательности двух слоев. Как показано в [14], доминирующим фактором в деформации ячейки могут оказаться механизмы роста. Поэтому в настоящей работе при создании многослойных гетероструктур использовался механизм роста по Франку-Ван дер Мерве, который идентифицировался по морфологии поверхности методами растровой микроскопии и просвечивающей электронной микроскопии высокого разрешения на микроскопах Tecnai G2 и FEI Titan 80-300.

\section{2. Эксперимент}

Многослойные гетероструктуры BST08/BST04/MgO и BST04/BST08/MgO на (100) MgO создавались высокочастотным распылением керамических мишеней на двух установках „Плазма СЭ50“. Установлено, что при прерывистом осаждении гетероэпитаксиальный рост различных составов продолжается без ухудшения структур- 
ного совершенства. Принципиальное отличие данного способа осаждения от известных аналогов состоит в том, что рост монокристаллических пленок происходит из дисперсной фазы оксида, образующейся в плазме сильноточного высокочастотного разряда при распылении керамической мишени на кластерном уровне [15]. Показано, что энергетическое состояние дисперсной фазы наиболее адекватно отображается обобщенным параметром (b) (энергетика пучковых электронов), количественное определение которого проводилось на основе оптической эмиссионной спектроскопии. Механизмами роста, степенью структурного совершенства, а тем самым и свойствами пленок можно широко варьировать, находясь в рамках трехмерного фазового пространства $b, p, q$, где $q-$ степень структурного совершенства пленки, $p$ - давление кислорода. Таким образом, впервые многопараметрическую задачу условий получения пленок удалось свести к одной фазовой диаграмме, что существенно упрощает создание гетероструктур по заданному механизму роста (по ФранкуВан дер Мерве или по Вольмера-Вебера).

Ранее $[16,17]$ нами было установлено, что параметры элементарной ячейки $a$ и $c$ пленок BST на (001)MgO, выращенных по Франку-Ван дер Мерве, в области толщин пленок от 6 до $70 \mathrm{~nm}$ изменяются незначительно, причем параметр $a$ больше, чем параметр $c$, что связано с присутствием в пленках двумерных растягивающих напряжений в плоскости подложки. В области толщин пленок, превышающих $70 \mathrm{~nm}$, происходит изменение знака напряжений в пленках: двумерные растягивающие напряжения в плоскости подложки изменяются на сжимающие. Это позволяет исследовать особенности проявления сегнетоэлектрического состояния в пленках наноразмерной толщины, в которых реализуются растягивающие либо сжимающие двумерные напряжения для одной системы пленка-подложка.

В настоящей работе исследовались двухслойные гетероструктуры BST08/BST04/MgO и BST04/BST08/MgO при толщине каждого слоя меньше $70 \mathrm{~nm}$ для реализации двумерных напряжений растяжения [9]. Толщина пленок определялась по времени напыления и уточнялась по высоте ступеньки после удаления части пленки BST, измеренной на атомном силовом микроскопе „Интегра“ фирмы НТ-МДТ. Структурное совершенство двухслойных структур, параметры элементарной ячейки в направлении нормали к плоскости подложки и вдоль плоскости подложки, а также эпитаксиальные соотношения между слоями пленки и подложкой при комнатной температуре устанавливались нами рентгенографиро-

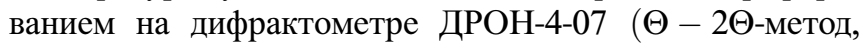
съемка симметричных и асимметричных брэгговских отражений). При описании результатов исследования нами выбрана координатная система, в которой нормальная к поверхности пленки ось обозначена как ось $c$, оси $a$ и $b$ располагаются в плоскости подложки. Параметры элементарной ячейки рассчитывались в орторомбическом приближении. Разделение перекрывающихся линий про- изводилось с помощью функции Войта. Линейная плотность дислокаций $(\rho)$ на границе раздела между пленкой и подложкой или на границе раздела между двумя слоями рассчитывалась по формуле $\rho=\left(a-a_{s}\right) /\left(a \cdot a_{s}\right)$, где $a_{s}$ - параметр решетки подложки или нижнего слоя. Для $\mathrm{MgO} a_{s}=0.4211 \mathrm{~nm}$. Деформация решетки $(\eta)$ определялась по формуле $\eta=\left(a-a_{0}\right) / a_{0}$, где $a_{0}$ - псевдо-кубический параметр решетки объемного материала. Для BST04 $a_{0}=0.3942 \mathrm{~nm}$, а для BST08 $a_{0}=0.3982 \mathrm{~nm}$.

Для изучения диэлектрических характеристик на поверхности гетероструктуры методами взрывной литографии формировались планарные электроды в форме встречно-штыревого преобразователя (ВШП) с алюминиевыми электродами $(300 \mathrm{~nm})$ и ванадиевым подслоем $(40 \mathrm{~nm})$. Взрывная литография обеспечивала отсутствие металла во всем технологическом цикле изготовления ВШП и исключала изменение поверхности гетероструктуры при удалении металла, необходимом для обычной литографии. Расстояние между штырями составляло $2 \mu \mathrm{m}$ при их количестве 76 шт. с перекрытием электродов $318 \mu \mathrm{m}$. Емкость, проводимость и токи утечки планарных конденсаторов измерялись на измерителе параметров полупроводникового прибора Keihtley 4200SCS на частоте $1 \mathrm{MHz}$ при помощи зондовой станции РМ-5 MicroTec.

Для исследования динамики переключения поляризации при различных амплитудах переменного пилообразного поля (от 0.5 до $300 \mathrm{kV} / \mathrm{cm}$ ) в диапазоне частот $\left(10^{-3}-10^{4}\right) \mathrm{Hz}$ была использована модифицированная методика Сойера-Тауэра. Для обеспечения высокого входного сопротивления, необходимого для работы на инфранизких частотах, сигнал с эталонного конденсатоpa $\left(\mathrm{C}_{0}=1.0 \mu \mathrm{C}\right)$ подавался на согласующий усилитель с высоким входным сопротивлением и единичным коэффициентом усиления. Сигнал воздействия с генератора через делитель напряжения и сигнал поляризации, снимаемый с выхода согласующего усилителя, подавались на два независимых аналого-цифровых канала платы сбора данных. Для независимого ввода и оцифровки сигналов с входов АЦП применялись два раздельных потока в программной реализации драйвера платы сбора данных. Это позволяло проводить независимо измерения, как полевого воздействия, так и поляризационного сигнала от времени [16].

\section{3. Результаты и обсуждение}

Исследование возникновения дислокаций несоответствия в гетероструктурах $\mathrm{MgO} / \mathrm{BST}$ и их изменения с толщиной проводилось методами микроскопии высокого разрешения [17]. Установлено, что при толщине пленок BST08 менее $6 \mathrm{~nm}$ дислокации несоответствия находятся выше границы раздела пленка-подложка и равномерно распределены по толщине пленки с расстояниями между дислокациями $4 \mathrm{~nm}$. С увеличением толщины пленки они становятся более упорядоченными и, начиная с $10 \mathrm{~nm}$, 
располагаются только на границе раздела, образуя дислокационную сетку с периодом примерно $12 \mathrm{~nm}$. Расчетный период следования дислокаций несоответствия с учетом параметров решеток для объемных материалов BST и $\mathrm{MgO}$ составляет примерно $7.3 \mathrm{~nm}$. Для пленок толщиной более $70 \mathrm{~nm}$ период следования дислокаций резко изменяется и составляет $3.6 \mathrm{~nm}$, т.е. меньше расчетного периода, что свидетельствует о напряжениях сжатия в пленке. Таким образом, электронная микроскопия высокого разрешения позволила подтвердить данные рентгеноструктурного анализа о наличии в пленках толщиной меньше $70 \mathrm{~nm}$ двумерных деформационных полей растягивающего типа. Естественно, для гетероструктур $\mathrm{MgO} / \mathrm{BST}$ изменение двумерного растяжения на двумерное сжатие приводит к существенным изменениям свойств [18].

Для всех исследованных двухслойных гетероструктур $\mathrm{MgO} / \mathrm{BST} 08 / \mathrm{BST} 04$ и $\mathrm{MgO} / \mathrm{BST} 04 / \mathrm{BST} 08$ (толщина каждого слоя $40 \mathrm{~nm}$ ) по данным $\varphi$-сканирования асимметричных рефлексов (113) и (103) слоев пленки и подложки наблюдалась полная параллельная ориентация как между слоями, так и с подложкой. Вертикальная разориентировка слоев пленки составляла $\sim 0.6^{\circ}$. Анализ полуширин асимметричных отражений от слоев пленки, записанных при неподвижных счетчике и образце при вращении структуры вокруг нормали к плоскости роста, показал, что азимутальная разориентировка составляет менее $0.5^{\circ}$.

Рентгенодифракционное исследование гетероструктур $\mathrm{MgO} / \mathrm{BST} 08, \mathrm{MgO} / \mathrm{BST} 04, \mathrm{MgO} / \mathrm{BST} 08 / \mathrm{BST} 04$ и $\mathrm{MgO} / \mathrm{BST} 04 / \mathrm{BST} 08$, выполненное при комнатной температуре, выявило наличие особенностей в параметpax элементарных ячеек пленок. Фрагменты рентгенограмм приведены на рис. 1. При комнатной температуре объемные образцы BST08 имеют тетрагональную симметрию с параметрами элементарной ячейки $a=0.3978 \mathrm{~nm}, c=0.3991 \mathrm{~nm}$, а ВST04 - кубическую $a=b=c=0.3942 \mathrm{~nm}$. Параметры элементарной ячейки пленки BST в направлении, перпендикулярном подложке, в однослойных гетероструктурах $\mathrm{MgO}$ /BST04 (рис. $1, a$ ) и $\mathrm{MgO} / \mathrm{BST08}$ (рис. $1, b$ ) равны соответственно 0.3945 и $0.3988 \mathrm{~nm}$. Параметр элементарной ячейки в плоскости подложки для BST04 $a=b=0.3952 \mathrm{~nm}$, а для BST08 $a=b=0.4001 \mathrm{~nm}$, т.е. больше, чем соответствующие параметры $c$ по нормали к подложке. Деформация решетки и линейная плотность дислокаций на границе раздела между пленкой и подложкой для BST04 равны $\eta=2.5 \cdot 10^{-3}, \rho=1.6 \cdot 10^{6} \mathrm{~cm}^{-1}$, а для BST08 $\eta=4.8 \cdot 10^{-3}, \rho=1.25 \cdot 10^{6} \mathrm{~cm}^{-1}$. Таким образом, в однослойных гетероструктурах $\mathrm{MgO} / \mathrm{BST} 04$ и MgO/BST08 присутствуют двумерные напряжения растяжения. Пленка BST04 находится в парафазе, а пленка BST08 в сегнетофазе, в которой вектор спонтанной поляризации лежит в плоскости подложки (a $а$-фаза). Для пленки BST08 температура перехода из парафазы в сегнетофазу, по данным рентгеноструктурного анализа, происходит при температуре $\sim 490 \mathrm{~K}$. Детальное рассмотрение фазовых

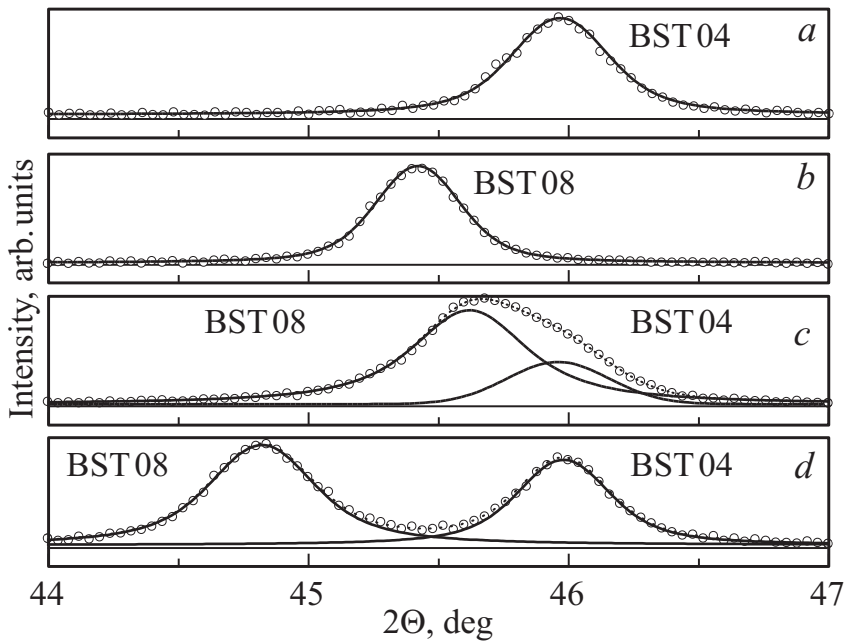

Рис. 1. Фрагменты рентгенограмм с экспериментальным профилем рефлекса (002) (кружочки) и разложение профиля на составляющие контуры (сплошные линии) гетероструктур: $a-\mathrm{MgO} / \mathrm{BST} 04, b-\mathrm{MgO} / \mathrm{BST} 08, c-\mathrm{MgO} / \mathrm{BST} 08 / \mathrm{BST} 04$ и $d-\mathrm{MgO} / \mathrm{BST} 04 / \mathrm{BST} 08$.

состояний для однослойных структур BST08 проведено в наших работах $[19,20]$.

На основе анализа рефлексов (002) от гетероструктуры $\mathrm{MgO} / \mathrm{BST} 08 / \mathrm{BST04}$ (рис. 1,c) установлено, что для нижнего слоя BST08 параметры элементарной ячейки равны $c=0.3974 \mathrm{~nm}$ и $a=b=0.4006 \mathrm{~nm}$, деформация решетки и линейная плотность дислокаций на границе раздела между пленкой BST08 и подложкой равны $\eta=6.0 \cdot 10^{-3}, \rho=1.22 \cdot 10^{6} \mathrm{~cm}^{-1}$. Это свидетельствует о том, что в двухслойной гетероструктуре $\mathrm{MgO} / \mathrm{BST} 08 / \mathrm{BST} 04$ двумерные напряжения растяжения в слое BST08 больше, чем в однослойной пленке BST08. Для пленки BST08 температура перехода из парафазы в сегнетоэлектрическую $a а$-фазу происходит при температуре $T \sim 520 \mathrm{~K}[20]$, т. е. выше, чем для однослойной пленки. Для верхнего слоя BST04 параметры решетки $c=0.3947 \mathrm{~nm}$ и $a=b=0.3938 \mathrm{~nm}, \eta=-1.0 \cdot 10^{-3}$, $\rho=0.42 \cdot 10^{6} \mathrm{~cm}^{-1}$, т.е. в этом слое присутствуют напряжения сжатия.

При изменении порядка чередования слоев $\mathrm{MgO} /$ BST04/BST08 (рис. $1, d$ ) деформация элементарной ячейки каждого слоя существенно изменяется по сравнению с гетероструктурой $\mathrm{MgO} / \mathrm{BST} 08 / \mathrm{BST} 04$. Для нижнего слоя BST04 параметры элементарной ячейки равны $c=0.3947 \mathrm{~nm}$ и $a=b=0.3953 \mathrm{~nm}, \eta=2.7 \cdot 10^{-3}$, $\rho=1.5 \cdot 10^{6} \mathrm{~cm}^{-1}$. Это свидетельствует о том, что в двухслойной гетероструктуре $\mathrm{MgO} / \mathrm{BST} 04 / \mathrm{BST} 08$ в слое BST04 присутствуют двумерные напряжения растяжения. Для верхнего слоя BST08 в этой двухслойной структуре $c=0.4039 \mathrm{~nm}$ и $a=b=0.3955 \mathrm{~nm}$, $\eta=-6.7 \cdot 10^{-3}, \rho=1.5 \cdot 10^{6} \mathrm{~cm}^{-1}$ присутствуют двумерные напряжения сжатия. По данным рентгеноструктурного анализа [20], при такой величине двумерных напряжений сжатия в слое BST08 при температуре 


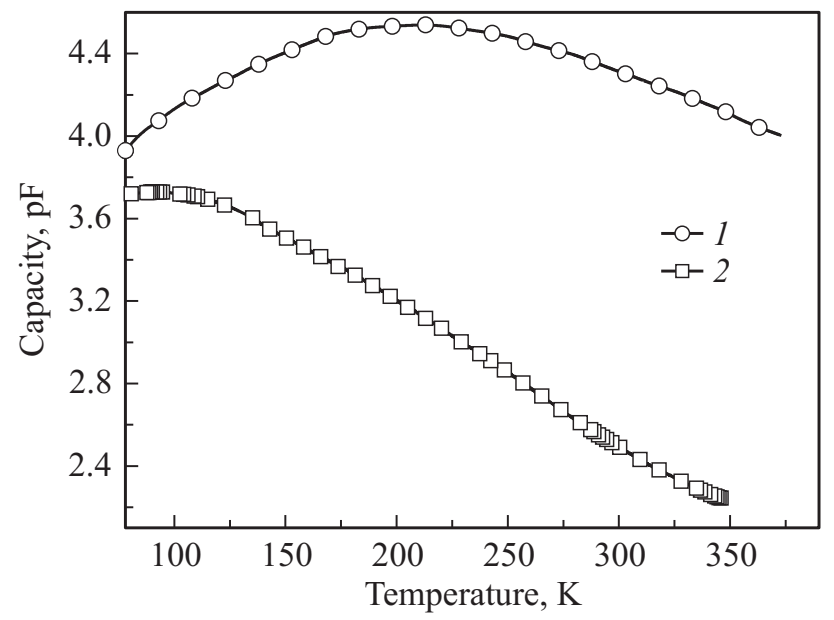

Рис. 2. Температурные зависимости емкости двухслойных гетероструктур в зависимости от порядка чередования слоев: 1 - MgO/BST08/BST04, 2 - MgO/BST04/BST08.

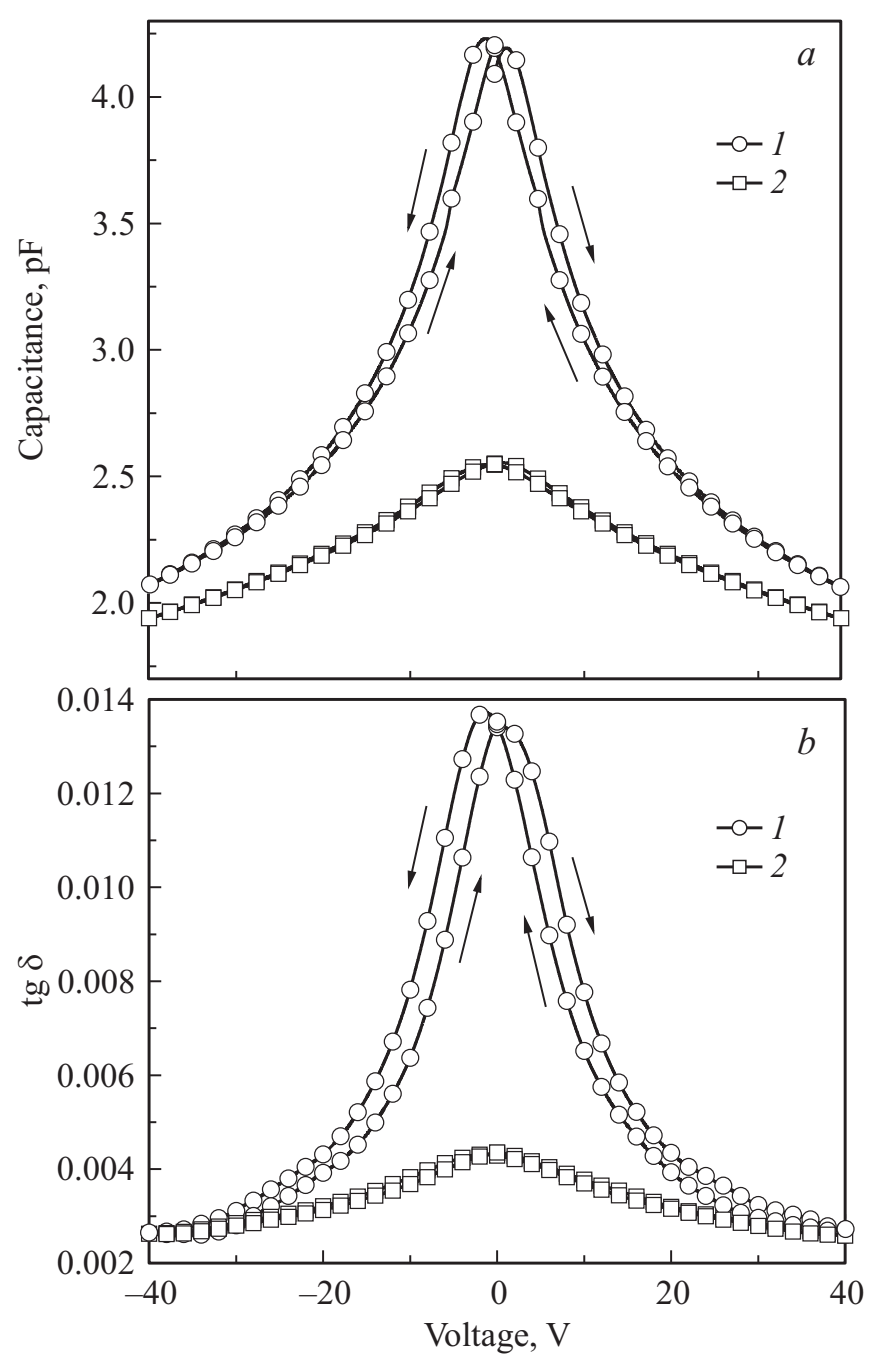

Рис. 3. Зависимости емкости (a) и тангенса потерь (b) от внешнего полевого воздействия двухслойных гетероструктур при различном порядке чередования слоев: $1-\mathrm{MgO} /$ BST08/BST04, 2 - MgO/BST04/BST08.

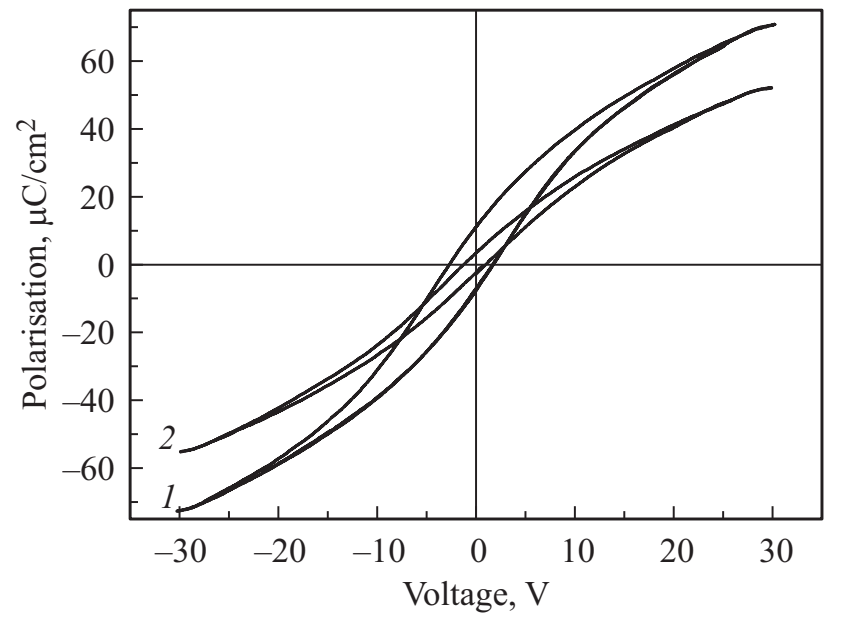

Рис. 4. Зависимость поляризации от внешнего полевого воздействия при температуре $77 \mathrm{~K}$ при различном чередовании слоев: 1 - MgO/BST08/BST04, 2 - MgO/BST04/BST08.

$T \sim 550 \mathrm{~K}$ происходит переход из парафазы в сегнетоэлектрическую $c$-фазу, в которой вектор спонтанной поляризации направлен вдоль нормали к подложке.

Таким образом, две гетероструктуры $\mathrm{MgO} / \mathrm{BST} 08 /$ BST04 и MgO/BST04/BST08 имеют качественные различия фазовых состояний в слоях BST08 при комнатной температуре. Такое различие должно существенно изменить диэлектрические характеристики.

Измерения зависимости диэлектрических характеристик структур от температуры показали, что температура фазового перехода из параэлектрической в сегнетоэлектрическую фазу для гетероструктуры (MgO/BST04/BST08) на 120 градусов ниже, чем в (MgO/BST08/BST04) и равна $448 \mathrm{~K}$ (рис. 2). Диэлектрическая управляемость (изменение диэлектрической проницаемости под действием внешнего электрического поля) в структуре $\mathrm{MgO} / \mathrm{BST0} / \mathrm{BST04}$ в два раза выше, чем в $\mathrm{MgO} / \mathrm{BST} 04 / \mathrm{BST} 08$, при диэлектрических потерях на уровне 0.01 (рис. 3 ).

При исследовании переполяризации гетеростуктур установлено, что форма петли диэлектрического гистерезиса при температуре $77 \mathrm{~K}$ не зависит от частоты во всем диапазоне $\left(10^{-3}-10^{4}\right) \mathrm{Hz}$ в пределах ошибки измерений. На рис. 4 приведены петли диэлектрического гистерезиса, измеренные на частоте $5 \mathrm{~Hz}$. Видно, что реориентационная поляризация при изменении чередования слоев в гетероструктурах $\mathrm{MgO} / \mathrm{BST} 04 / \mathrm{BST} 08$ и $\mathrm{MgO} / \mathrm{BST} 08 / \mathrm{BST} 04$ составляет 25 и $20 \mu \mathrm{C} / \mathrm{cm}^{2}$, а остаточная поляризация равна 5 и $10 \mu \mathrm{C} / \mathrm{cm}^{2}$ соответственно.

\section{4. Заключение}

В зависимости от порядка следования слоев BST различного состава в двухслойных гетероструктурах $\mathrm{MgO}+\mathrm{Ba}_{0.8} \mathrm{Sr}_{0.2} \mathrm{TiO}_{3}+\mathrm{Ba}_{0.4} \mathrm{Sr}_{0.6} \mathrm{TiO}_{3}$ происходит усиле- 
ние двумерного растягивающего напряжения в прилегающем к подложке слое (не зависимо от чередования слоев) по сравнению с однослойными гетероструктурами. В верхних же слоях BST04 или BST08 присутствуют двумерные напряжения сжатия. Отмеченные эффекты определяются нелинейным взаимодействием слоев между собой и подложкой, которое приводит к изменению симметрии решетки ( $a a$-фаза), а также влиянием доменной структуры, формирующейся после осаждения $\mathrm{Ba}_{0.8} \mathrm{Sr}_{0.2} \mathrm{TiO}_{3}$ в качестве первого слоя. Это проявляется в образовании дополнительной сетки дислокаций на границах двух сегнетоэлектрических слоев по сравнению с однослойными гетероструктурами.

Таким образом, использование двухслойных гетероструктур открывает новые возможности для управления диэлектрическими свойствами гетероструктур, что важно при использовании их в качестве электрически управляемых устройств сверхвысокочастотного и оптического диапазонов. С практической точки зрения интересен факт повышения термостабильности диэлектрической проницаемости на уровне 2\% в интервале температур от -50 до $50^{\circ} \mathrm{C}$ для гетероструктуры (MgO/BST08/BST04) при сохранении высокой диэлектрической управляемости $n=C_{0} / C_{V=40 \mathrm{~V}}>2$. При изменении чередования слоев $\operatorname{tg} \delta$ понижается до значения 0.004 при $n=C_{0} / C_{V=40 \mathrm{~V}}>1.5$.

Следует отметить, что при статическом электрическом поле $20 \mathrm{~V} / m u$ т значения емкости и $\operatorname{tg} \delta$ структур принимают одинаковые значения для гетероструктур с различным чередованием исследуемых составов. Этот факт может свидетельствовать о нивелировании диэлектрических параметров структур в сильном электрическом поле, первоначально различающихся из-за различных внутренних механических напряжений.

\section{Список литературы}

[1] S. Corkovic, Q. Zhanga. J. Appl. Phys. 105, 061610 (2009).

[2] M.W. Cole, E. Ngo, C. Hubbard, S.G. Hirsch, M. Ivill, W.L. Sarney, J. Zhang, S.P. Alpay. J. Appl. Phys. 114, 164107 (2013).

[3] Y. Lin, C.L. Chen. J. Mater. Sci. 44, 5274 (2009).

[4] H.N. Lee, H.M. Christen, M.F. Chisholm, C.M. Rouleau, D.H. Lowndes. Nature. 433, 395 (2005).

[5] M. Tyunina, I. Jaakola, M. Plekh, J. Levoska. J. Electroceram 24, 15 (2010).

[6] M. Liu, C. Ma, G. Collins, J. Liu, C. Chen, A.D. Alemayehu, G. Subramanyam, Y. Ding, J. Chen, C. Dai, Y.L. Me W Cole. Nanoscale Res. Lett. 8, 338 (2013).

[7] B.D. Qu, M. Evstigneev, D.J. Johnson, R.H Prince. Appl Phys. Lett. 72, 1394 (1998).

[8] B. Misirlioglu, G. Akcay, S. Zhong, S.P. Alpay. J. Appl. Phys. 101, 036107 (2007).

[9] T.Q. Ngo, A.B. Posadas, M.D. McDaniel, C. Hu, J. Bruley, E.T. Yu, A.A. Demkov, J.G. Ekerdt. Appl. Phys. Lett. 104, 082910 (2014).

[10] I. Jaakolaa, J. Levoska, M. Tyunina. Ferroelectrics 335, 127 (2006).
[11] F. Xue, J.J. Wang, G. Sheng, E. Huang, Y. Cao, H.H. Huang, P. Munroe, R. Mahjoub, Y.L. Li, V. Nagarajan, L.Q. Chen. Acta Mater. 61, 2909 (2013).

[12] J.H. Haeni, P. Irvin, W. Chang, R. Uecker, P. Reiche, Y.L. Li, S. Choudhury, W. Tian, M.E. Hawley, B. Craigo, A.K. Tagantsev, X.Q. Pan, S.K. Streiffer, L.Q. Chen, S.W. Kirchoefer, J. Levy, D.G. Schlom. Nature 430, 758 (2004).

[13] J. Wang, J.B. Neaton, H. Zheng, V. Nagarajan, S.B. Ogale, B. Liu, D. Viehland, V. Vaithyanathan, D.G. Schlom, U.V. Waghmare, N.A. Spaldin, K.M. Rabe, M. Wuttig, R. Ramesh. Science 299, 171 (2003).

[14] В.М. Мухортов, Ю.И. Головко, С.В. Бирюков, А.С. Анохин, Ю.И. Юзюк. ЖТФ 86, 93 (2016).

[15] В.М. Мухортов, Ю.И. Юзюк. Гетероструктуры на основе наноразмерных сегнетоэлектрических пленок: получение, свойства и применение. ЮНЦ РАН, Ростов н/Д (2008). $224 \mathrm{c}$.

[16] V.M. Mukhortov, Yu.I. Golovko, P.A. Zelenchuk, Yu.I. Yuzyuk. Integrated Ferroelectrics 107, 83 (2009).

[17] В.М. Мухортов, Ю.И. Головко, А.А. Маматов, О.М. Жигалина, А.Н. Кускова. ЖТФ 80, 77 (2010).

[18] В.М. Мухортов, Ю.И. Головко, С.В. Бирюков, А.С. Анохин, Ю.И. Юзюк. ЖТФ 86, 93 (2016).

[19] Ю.И. Головко, В.М. Мухортов, Ю.И. Юзюк, Р.Е. Janolin, B. Dkhil. ФTT 50, 467 (2008).

[20] P.-E. Janolin, A.S. Anokhin, Z. Gui, V.M. Mukhortov, Yu.I. Golovko, N. Guiblin, S. Ravy, M.El. Marssi, Yu.I. Yuzyuk, L. Bellaiche, B. Dkhil. J. Phys.: Condens. Matter 26, 292201 (2014). 